\title{
On-photo Restitution and Management of an Angular Size-lllusion's Behaviour Experienced in Architectural/Urban Spaces
}

\author{
Djordje DJORDJEVIC*, Mirjana DEVETAKOVIC RADOJEVIC, Nikola POPOVIC
}

\begin{abstract}
An angular size-illusion refers to a contradictory effect related to a seeming size-decrease of focused objects as the observer approaches them. This paper continues the research in this field, trying to establish fundamental principles of how to: (a) efficiently perform an on-photo restitution of an illusion's behaviour (expressed by illusion descriptors) when it is experienced in architectural/urban spaces during movement under the influence of available triggers (represented by illusion determinants of physical nature), and (b) manage it computationally so as to be sustainable for contemporary professional practice. To explain that conceptually, one simple architectural/urban matrix is chosen and digitally photographed. Images are photogrammetrically processed and determinants-related data obtained. Subsequently, descriptors-related outputs are calculated by applying derived mathematical equations (expressed in function of those determinants-related data). Then, behaviour-charts are created and corresponding illusion-characteristics read-off. Finally, it is illustrated how to manage (intentionally modify) the restituted behaviour by varying values of acquired determinants-related data. Given results allow also to "design a new illusion" (to programme and control it) by simulating in digital VR/AR environments. Thus, any cause of unwanted/unpredictable visual impression degradations of important architectural/urban structures can be prevented or minimized (by planning and performing adequate spatial/physical interventions on existing/reconstructed/newly designed matrices).
\end{abstract}

Keywords: angular size-illusion; architecture; augmented reality; photogrammetry; urbanism; virtual reality

\section{INTRODUCTION}

According to the law on perspective perception of three-dimensional spaces, as the observer moves towards the object of perception, the latter appears larger and viceversa. However, under some circumstances, as the distance changes, the impression of the perceived object's volume does not change as described - but inversely. As it has already been confirmed in [1], this phenomenon is triggered by the influence of an angular size-illusion exhibited during the observer's movement due to the impact of specific perceptual factors in the form of distance-depth cues as well as specific neural activities [2], including an additional influence of a perceptual phenomenon known as the oculomotor micropsia [3-5].

Contemporary architectural/urban knowledge and practice requires a permanent connection to other "edging" professions whereof the authors resumed this multidisciplinary scientific approach in a subject-related field of interest: they used again in [6] a necessary psychological knowledge to broaden fundamentals of that illusion, initially investigated in [1]. The results thereof enabled to scientifically describe the entire illusion's behaviour in reality, in a way applicable in contemporary architectural/urban theory and its everyday practice.

This paper continues such multidisciplinary approach by implementing practical contemporary photogrammetric knowledge to efficiently reach the research aim - to establish fundamental principles of how to: (a) efficiently perform an on-photo restitution of an illusion's behaviour (expressed by illusion descriptors) which is experienced in architectural/urban spaces during movement - under the influence of available triggers (represented by illusion determinants of physical nature), and (b) manage it numerically/computationally so that it is sustainable for actual professional practice. To explain this conceptually, one simple architectural/urban matrix is chosen and digitally photographed. By processing those images photogrametrically, relevant determinants-related data are acquired. Subsequently, descriptors-related outputs are calculated by using mathematical equations of algebraic and trigonometric nature (expressed in function of identified determinants-related data). Then, behaviourcharts are created and corresponding characteristics readoff [6]. Finally, it is illustrated how it is possible to manage (intentionally modify) the restituted behaviour by varying values of acquired determinants-related data.

The nature of the analyzed concept indicates that it can be successfully implemented notwithstanding whether the restitution procedure is performed automatically/semi automatically (photogrammetrically in this case) or manually (by applying a standard perspective-restitution technique). Evidently, the advantage of photogrammetry is that it makes possible to obtain the results more efficiently, because it is a less time-consuming and more accurate procedural activity (if and when needed).

Despite the way the on-photo restitution technique is performed in, this approach allows us to restitute the behaviour of an "existent" illusion or to "redesign" it in prospect (namely, to programme it by performing adequate physical interventions onto relevant architectural/urban masses). Additionally, the approach can be considered universal, because it ought to be applied in the following two cases as well: (a) when analyzed architectural/urban matrix parts (existent) have not been GIS-mapped yet, so to make available-to-process in the subject-related way their photo material mainly (as it will be described in this experiment) and (b) when analyzed architectural/urban matrix parts (currently partially or fully devastated) have to be reconstructed, primarily based on archived photo/video material (because relevant technical documentation is deficient, not available or not existent at all). With regard to the mentioned, using the investigated apparatus, each of spatial/physical interventions (necessary to perform either on existent or newly designed architectural/urban matrix parts) can be carefully planned, conducted and monitored from the subject-related point of view, in order to timely prevent or minimize any cause of probable unwanted/unpredictable visual impression degradations of built structures (especially those declared "a great importance").

So, the main contribution for the future is to regard the given results as starting points necessary to concretize in IT-sense a concept of completely automated/computer- 
guided illusion-designing (its programming and simulation) in virtual-reality and augmented-reality environments (thus fit to be broadly applicable not only in architecture/urbanism but in a variety of its "edging" domains).

\section{PREVIOUS SUBJECT-RELATED RESEARCH}

Numerous studies in optics, neurophysiology and psychology have investigated various types of optical/visual illusion, including the so-called angular size-illusion phenomenon, trying to reveal sustainable fundamentals that could explain when and why the visual sensory system alters the reading of existing characteristics of the observed visual field [7-19].

Angular size-illusion research closely connected to the field of architecture/urbanism, confirmed that the paradoxical visual effect related to a seeming size-decrease of architectural/urban objects - noticeable as the observer approaches them, can be considered a consequence of this angular size-illusion influence [1]. Subsequently, the same authors investigated in [6] the various aspects of impact of different architectural/urban pattern-configuration types on the illusion's behaviour - exploring, identifying and formulatinga set of factors of physical nature that influence it specifically (so-called illusion-determinants). Also investigated were fundamental behavioural characteristics of an exhibited illusion (expressed by so-called illusion-descriptors), suitable for simple mathematical (numerical) modelling.

Thus, in the actual same-scope study, the authors started to implement relevant photogrammetric technique (and corresponding software) to initially process subject-related data as efficiently as possible.

An indirect contribution of [20] to this work, is to better understand how the informative quality of perceived architectural/urban scenes (valorised by the presence of various constructor and destructor signals recognizable in different depth-planes) inevitably leads to dynamic changes of the observer's subjects of interest during movement (from close, to newly focused targets located remotely) and, thus, to a permanent reorganisation of the visual field (caused by a reorientation of the perception direction: with or without a head or eye movement and influenced by visual, selective and controlling attention mechanisms).

In common, in [21] was revealed the manner a built environment (or one in draft) - composed of existing (or planned) architectural/urban entities may change the observer's visual attention's mechanism (with or without the influence of additional educational training) and, therefore the choice of spatial entities which would be identified as pertinent entities-to-perceive (also essential to activate an angular size-illusion). Moreover, effects of environment on spatial knowledge (namely, of an angle between navigation paths and transitional spaces) were explained in [22], enriching hence the present, mainly, perceptually-oriented research by applying a cognition-related approach. In line with that and with the authors' previous subject-related research $[1,6]$, it is concluded in [23] too that another paradoxal response of the visual perception system (related to the hovering effect of perceived built structures) is also influenced by a set of cognition-related determinants of physical nature as triggering factors of such an illusion-wise perceptual experience.

\section{BASIC TERMS, SYMBOLS, DEFINITIONS AND UNITS USED}

Although the majority of terms used in this paper considered to be relevant and starting were already investigated in [1] and [6], their symbols and definitions/meanings should be briefly highlighted again. Their graphic representation is shown in Fig. 1.

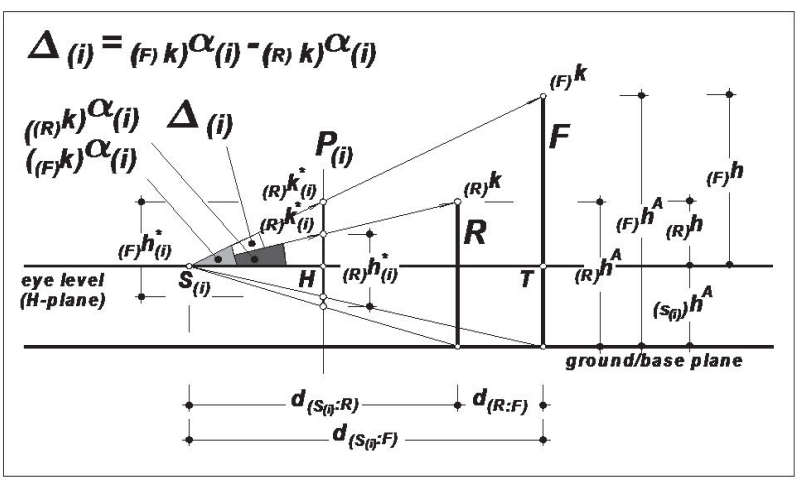

Figure 1 Graphic representation of subject-related parameters as well as their meanings (expressed by relevant linear and angular interrelations)

The starting terms used are as follows:

- $\quad F$ - Subject of perception (hereinafter: Focus) - an architectural/urban object focused (targeted) as an actual observer's subject of interest,

- $\quad T$ - Principal point (hereinafter: Target) -a central point of the observer's visual field (and, consequently the actual centre of a non-cropped photo), namely, the point of the façade of $F$ perceived by the observer as his primary subject of interest (identified as such by the influence of selecting and controlling visual attention's mechanisms $[20,21])$,

- $\quad R$ - Visual marker (hereinafter: Marker) - a referential spatial entity of various nature which, in a visually competitive relation with respect to $F$, makes the illusion possible during movement $[1,6]$; in this research, entity $R$ is an architectural object located in the surrounding of $F$,

- $\quad{ }_{(F)} k$ - Upper contour-line of the observed façade of $F$,

- $\quad{ }_{(R)} k$ - Upper contour-line of the observed façade of $R$, and

- $\quad S_{(i)}$ - Station-point - a camera's/observer's position on the movement-path, numbered as $i$ ( $i=1: m$, where $m$ is the total number of analyzed positions/restituted photos).

Since perspective pictures can be declared graphical representations of digital/analog photos, the following two terms ought to be newly introduced:

$P_{(i)}-$ Visual/graphic representation of the observer's visual field (in the form of photograph in this research), that corresponds to the location of $S_{(i)}$ (hereinafter: Image/perspective picture plane), and 
- $H$ - "Imaginary" line located in $P_{(i)}$ (hereinafter: Horizon) - an imaginary intersection line between $P_{(i)}$ and an imaginary horizontal plane (hereinafter: $H$-plane) that the focus of camera lens (namely, the observer's eye/optical axis) belongs to.

For clarity reason, out of the two illusion's determinants introduced in [6], a simpler one will be analyzed in this study too: metric difference between the heights of ${ }_{(F)} k$ and ${ }_{(R)} k$ (of the corresponding $F$ and $R$, respectively), including the distance between $F$ and $R$.

The other subject-related data-types and their symbols, names and definitions/meanings are given below (most of them are graphically presented in Fig. 1):

- $\quad{ }_{(F)} h^{A}$ - Actual height of $F$ (measured from its groundplane to $\left._{(F)} k\right)$,

- $\quad{ }_{(R)} h^{A}$ - Actual height of $R$ (measured from its groundplane to $\left._{(R)} k\right)$,

- $\quad{ }_{(F)} h$ - Height of $F$ (measured from $H$-plane to ${ }_{(F)} k$ ),

- $\quad{ }_{(R)} h$ - Height of $R$ (measured from $H$-plane to ${ }_{(R)} k$ ),

- $\quad{ }_{\left(S_{(i)}\right)} h^{A}$ - Actual height of $S_{(i)}$ (measured to the groundplane),

- $\quad(S) \bar{h}$ - Average height of station-points used,

- $d_{(R: F)}$ - Distance from $F$ to $R$,

- $d_{\left(S_{(i)}: F\right)}$ - Distance from $S_{(i)}$ to $F$,

- $d_{\left(S_{(i)}: R\right)}$ - Distance from $S_{(i)}$ to $R$,

- $d_{\left(S_{(i)}: S_{(i-1)}\right)}$ - Distance between two consecutive station-points: $S_{(i)}$ and $S_{(i-1)}$,

- $\bar{d}_{(S)}$ - Average distance between two consecutive station-points,

- ${ }_{(F)} k_{(i)}^{*}$ - Upper contour-line of the façade of $F$ perceived from $S_{(i)}$ (represented in $\left.P_{(i)}\right)$,

- ${ }_{(R)} k_{(i)}^{*}$ - Upper contour-line of the façade of $R$ perceived from $S_{(i)}$ (represented in $\left.P_{(i)}\right)$,

- $\quad{ }_{(F)} h_{(i)}^{*}$-Height of $F$ perceived from $S_{(i)}$ (represented in $P_{(i)}$ and measured to $\left.(F) k_{(i)}^{*}\right)$

- $\quad{ }_{(R)} h_{(i)}^{*}$-Height of $R$ perceived from $S_{(i)}$ (represented in $P_{(i)}$ and measured to $\left.{ }_{(R)} k_{(i)}^{*}\right)$,

- $\quad{ }_{\left.(F)^{k}\right)} \alpha_{(i)}$-Visual angle under which the height ${ }_{(F)} h$ is perceived from $S_{(i)}$,

- $\quad{ }_{\left({ }_{(R)} k\right)} \alpha_{(i)}$-Visual angle under which the height ${ }_{(R)} h$ is perceived from $S_{(i)}$.

Will also be used two illusion-quantifiers (already investigated in [6]), as subject-related:

- Delta-quantifier ((hereinafter: $\left.\Delta_{(i)}\right)$ - a core parameter used in ([1]) to describe an angular size-illusion's fundamental nature (by expressing the differences between visual angles $\left.{ }_{(F)} k\right) \alpha_{(i)}$ and ${ }_{\left({ }_{R}\right) k} \alpha_{(i)}$ under which $_{(F)} k$ and ${ }_{(R)} k$ are simultaneously perceived from the same $S_{(i)} /$ Fig. 1), and

- Psi-quantifier ((hereinafter: $\Psi_{(i \rightarrow(i-1))}$ - a quantifier of the illusion dynamics [6]; it expresses the level of delta-quantifier variation divided by the elapsed time of that variation and, thus, of $F$-to- $R$ dimensional seeming-change impression; given a non-temporal viewpoint, the psi-quantifier value can be reformulated metrically - as a value of delta-quantifiers variation divided by a distance between station-points that correspond to this variation (namely, by the distance between their two consecutives).

The common linear measurement unit (for lengths, heights and distances) will be metre, while the angular unit will be degree.

\section{SETUP OF THE EXPERIMENT \\ 4.1 Procedural Stages Definition}

Two procedural stages of the demonstrated illusionrestitution's concept ought to be performed consecutively to obtain aim-directed intermediate and final outputs.

Those stages are:

- Perspective restitution, namely, photogrammetric restitution in this research (hereinafter: $P R$ ), intended to identify: (a) fundamental metric characteristics of the analyzed urban matrix which are responsible for the illusion triggering and lasting (hereinafter: determinants-related data of pattern-morphogenic nature $([1,6]))$, and (b) spatial positions of each of $S_{(i)} \mathrm{s}$ the restituted photos were taken from and, consequently, their actual distribution along the entire movement-path, and

- "Angular" restitution (hereinafter: $A R$ ), intended to find the values of so-called "perceived angular sizes" of $F$ and $R$ [1] (based on output-data obtained by previously performed $P R$-procedure) and, subsequently, to find values of final numerical outputs in form of illusion quantifiers of delta- and psi-type. Doing so, it becomes possible to generate illusion's behaviour-charts and to read-off different aspects of that behaviour, such as: noticeability, appearance, duration, dynamics and overall impression [6].

\subsection{Equipment and Technology Used}

To realize the previously mentioned illusionrestitution stages, the following equipment is applied:

- Computer: Acer Aspire Timeline X with IntelCore 5 45DM processor and 8GB DDR3 RAM;

- Professional photogrammetric software, produced by EosSystems Inc: PhotomodelerPremium Ver. 2020/ 64-bit (hereinafter: PMP);

- Semi-professional 8MP camera: PowerShot S5 IS, produced by Canon Corp. (sensor format size (in $\mathrm{mm}$ ) is 5,726/4,293, while (in pxls) it is $3,264 / 2,448)$, and 
- Microsoft Excel Ver. 2003 - used for the final mathematical calculations and, consequently, behaviour-charts creation.

\subsection{Assumptions}

Having in mind the aim of this research (to demonstrate the illusion-restitution technique conceptually and comprehensively), two classes of assumptions will be tasked without deeper elaboration: (a) general constraints defined to choose an adequate experimental scene and to properly conduct the experiment as simple as possible (from the restitution-wise point of view), and (b) prerequisites related to technical aspects of the $P R$-procedure that refer to the close-range photogrammetric processing itself.

\subsubsection{General Constraints}

Considering the aforementioned, several general constraints ought to be pointed-out [1]:

- Concerning 3D-pattern morphology (geometry):

(a) both $F$ and $R$, including the movement-path, should be positioned onto the same horizontal ground-plane, (b) the perceived façades of $F$ and $R$ should be almost "frontal" ("frontal" means perpendicular to the observer's movement-path - authors' remark), flat and mutually parallel, (c) movement-path should be almost straight-lined and positioned onto the vertical symmetry-plane of $F$ as much as possible, and (d) the perceived $_{(F)} k$ and $_{(R)} k$ (of corresponding $F$ and $R$, respectively), should be also almost horizontal and straight-lined,

- Concerning movement: (a) camera/observer should move permanently towards $F$ - along symmetry-axes of the selected movement-path, and (b) $S_{(i)}$ s should be distributed along the movement-path uniformly, for clarity reason only (namely, to be mutually equidistant as much as possible), and

- Concerning observing/photo-shooting: (a) all $S_{(i)}$ s should nearly have the same height, and (b) shooting/perception direction (an optical axis) should be horizontal and permanently directed towards $F$ during the entire movement (so that $T$ remains unchanged).

\subsubsection{Prerequisites of Technical Nature}

The major $P R$-related prerequisites of technical nature ought to be also concisely considered [24-26]. They are organized into several groups, depending on the aspects they refer to:

- Regarding field-preparation and atmospheric conditions: using an appropriate number of adequate target-types properly distributed in the scene, choosing a proper day-time with respect to the recommended day-light characteristics and shadow-distribution,

- Regarding shooting procedure: taking a recommended number of photos, having in mind proper shooting-angle separations, photo-coverage and overlapping percentage of consecutive images,
- Regarding camera-presets: selecting a recommended image-resolution, focal length of camera lens, aperture (depth-of-field-related), shutterSpeed, ISO-speed, including settings that must be turned off (auto-focus, digital zoom, image stabilization, auto-sharpening and image-rotation),

- Regarding photogrammetric data-processing: using a sub-pixel marking tool (if any), fulfilling recommended settings of camera calibrator as well as of photo-matching, photo-referencing and photoorienting procedures, and

- Regarding photos post-processing: respecting the key fact that several image-editing activities must not be performed at all (such as image cropping, aspectratio changing, perspective rectifying), while image resampling, brightness, saturation and contrast modifying must not be performed partially and inadvertently.

\subsection{Mathematical Equations Definition}

Two groups of restitution-wise mathematical equations are derived therein with regard to procedural stages to apply to.

Their final forms are given below:

$\boldsymbol{P R}$-equations - intended to find relevant numerical outputs of the entire $P R$-procedure:

$\bar{d}_{(S)}==\frac{\sum_{i=1}^{m-1}\left(d_{\left(S_{(i+1)}: F\right)}-d_{\left(S_{(i)}: F\right)}\right)}{2 \times(m-1)}+$

$+\frac{\sum_{i=1}^{m-1}\left(d_{\left(S_{(i+1)}: R\right)}-d_{\left(S_{(i)}: R\right)}\right)}{2 \times(m-1)}$

where $m$ is a total number of $S_{(i)}$ s positioned along the observer's movement-path

${ }_{(S)} \bar{h}=\frac{\sum_{i=1}^{m}\left(_{\left(S_{(i)}\right)} h^{A}\right)}{m}$

where $m$ is a total number of $S_{(i)}$ s positioned along the observer's movement-path

$\boldsymbol{A R}$-equations - intended to find relevant numerical outputs of the $A R$-procedure, including the final results of the entire restitution of the illusion's behaviour.

${ }_{(F)} h={ }_{(F)} h^{A}-(S) \bar{h}$

${ }_{(R)} h={ }_{(R)} h^{A}-{ }_{(S)} \bar{h}$

Also, the following mathematical equations are derived by adapting corresponding ones given in [1] and [6]: 


$$
\begin{aligned}
& \left.{ }_{(F)} k\right) \alpha_{(i)}=\arctan \left[\frac{{ }_{(F)} h}{d_{\left(S_{(i)}: F\right)}}\right] ; \\
& \left.{ }_{(R)} k\right) \alpha_{(i)}=\arctan \left[\frac{{ }_{(R)} h}{d_{\left(S_{(i)}: R\right)}}\right] \rightarrow \\
& \Delta_{(i)}={ }_{\left({ }_{(F)} k\right)} \alpha_{(i)}-{ }_{\left({ }_{(R)} k\right)} \alpha_{(i)} \rightarrow \\
& \Delta_{(i)}=\arctan \left[\frac{{ }_{(F)} h \times d_{\left(S_{(i)}: R\right)}-{ }_{(R)} h \times d_{\left(S_{(i)}: F\right)}}{d_{\left(S_{(i)}: R\right)} \times d_{\left(S_{(i)}: F\right)}+{ }_{(F)} h \times{ }_{(R)} h}\right] \\
& \Delta_{(i)}=\arctan \left[\frac{(F)}{d_{\left(S_{(i)}: R\right)} \times d_{\left(S_{(i)}: R\right)}-{ }_{(R)} h \times\left(d_{\left(S_{(i)}: R\right)}+d_{(R: F)}\right)_{(F)} h \times{ }_{(R)} h}\right] \\
& \left.\Psi_{(i \rightarrow(i-1))}=\frac{\Delta_{(i)}-\Delta_{(i-1)}}{\bar{d}_{(S)}}\right)
\end{aligned}
$$

\subsection{Study-case Definition: Urban-matrix Selection}

Taking into account general constraints listed in Subsection 4.3.1 (conformed with general triggering conditions of the subject-related illusion [1]), an adequate architectural/urban location in the city of Belgrade/Serbia is chosen. This research will analyze a horizontal straight-lined segment of Kralja Milana Street: located from Kneza Milosa Street to Slavija Square. Due to its simplicity in morphological (geometric) sense and, thus, an ability to demonstrate the illusion-restitution concept illustratively, that location is declared an experimental field (hereinafter: $E F$ ). Shown in Figs. 2a, 2b, 2c is a triptych of $E F$ shots selected amongst those numerous capable to clearly demonstrate an exhibited illusion to a third-party who has not experienced it yet in reality.

In that triptych, $F$ is St. Sava Temple (a remote, centrally-positioned object), while the perceived volume of $R$ is defined in reality by the surrounding buildings and vegetation both visually competitive with respect to $F$. The $R$ 's volume parts of a built nature consist of visually dominant left- and right-sided architectural objects (represented by the Old -and New "Slavija" hotels). As the height of the right-sided $R$ 's part (i.e. the height of New Slavija Hotel) is visible from each of the analyzed $S_{(i)}$ s (contrary to that on the left-side), that $R$ 's part is the chosen reference.

These representative photos are taken from the $S_{(i)} \mathrm{s}$ located: far from the corresponding $F$ (when it is seemingly perceived as higher than $R$ - Fig. 2a, at the first fifth of the movement-path (when $F$ catches up in height with $R$ - Fig. $2 \mathrm{~b}$, and close to the corresponding $F$ (when it is seemingly perceived as lower than $R$ - Fig. 2c.

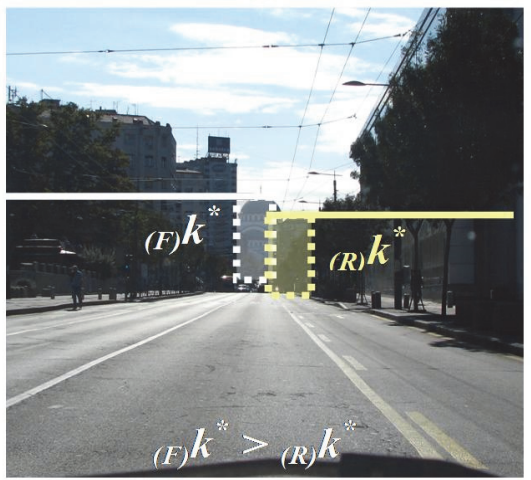

(a)

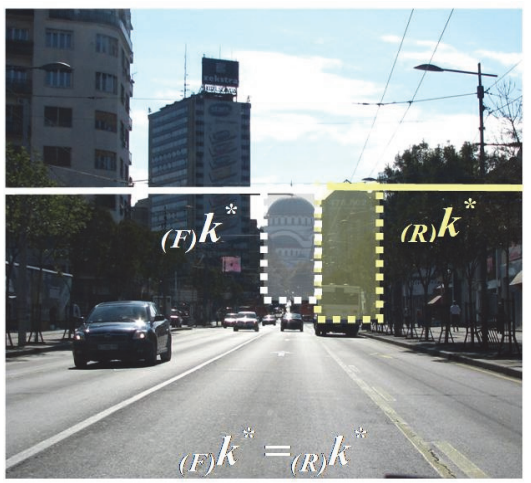

(b)

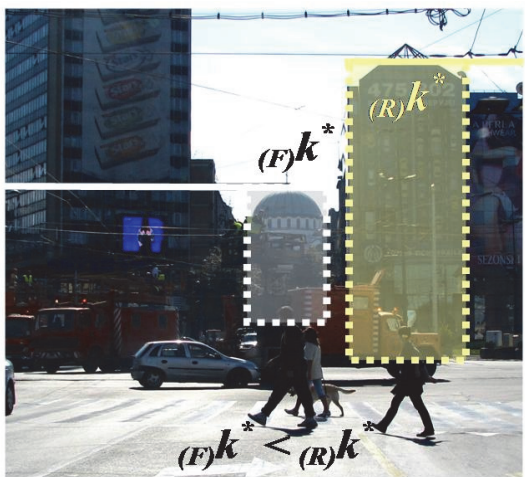

(c)

Figure $2 \mathrm{~A}$ triptych of representative shots of selected $E F$ chosen to illustrate an angular size-illusion phenomenon noticeable during the observer's movement towards the corresponding $F$ (expressed as an evident dimensional seeming-change impression between their heights): (a) camera is positioned far from $F$ (when it is seemingly perceived as higher than $R$ ); (b) camera is positioned at the first fifth of the movement-path (when $F$ catches up in height with $R$ ) and (c) camera is positioned near to $F$ (when it is seemingly perceived as lower than $R$ )

\section{REVIEW OF PROCEDURAL STEPS \\ 5.1 PR-procedure \\ 5.1.1 Fundamentals}

Bearing in mind the assumptions tasked in Sub-section 4.3, EF is digitally photographed from fifty $S_{(i)}$ s of approx. equal heights, so that ten of them be distributed along the movement-path: from the starting position (Kneza Milosa St.) to the ending position (Slavija Square, exactly: Beogradska St.), attempting at being mutually equidistant as much as possible. Also, shooting directions corresponding to these ten locations are directed towards $T$, while others are chosen with respect to technical (photogrammetric-related) prerequisites listed in Sub-section 4.3.2.
After shooting, the initial photogrammetric processing of the entire set of taken photos (that is their initial automatic point-marking and referencing intended to self-calibrate/solve the camera and, thus, to orient the photos with perspective deformations rectified) was performed by running a so-called PMP Smart-match procedure. It could have been realized due to the fact that $E F$ is full with so-called "natural targets". Because of the conceptual aim of the research where accuracy of the achieved photogrammetric results is not a point of interest, such target-type could have been used instead of printed large-format $R A D$ - and $D O T$-targets (needed to be properly distributed throughout the entire/large $E F$ before shooting).

Subsequently, due to that "conceptuality", not the entire $E F$ was photogrammetrically processed (modelled) 
but only its entities primarily relevant from the subject-related point of view (those of pattern-morphogenic nature as an illusion's determinants-related data). Such a model, as simplified representation of $E F$, is declared experimental model (hereinafter: $E M$ ). For the same reason, that $E M$ is exclusively numerically defined (not structurally - in form of 3D-mesh).

One of the ten "on-path" shots is shown in Fig. 3. This photo is arbitrarily chosen to graphically represent previously mentioned $E F$ elements (as relevant entries for its subsequent photogrammetric dimensional restitution).

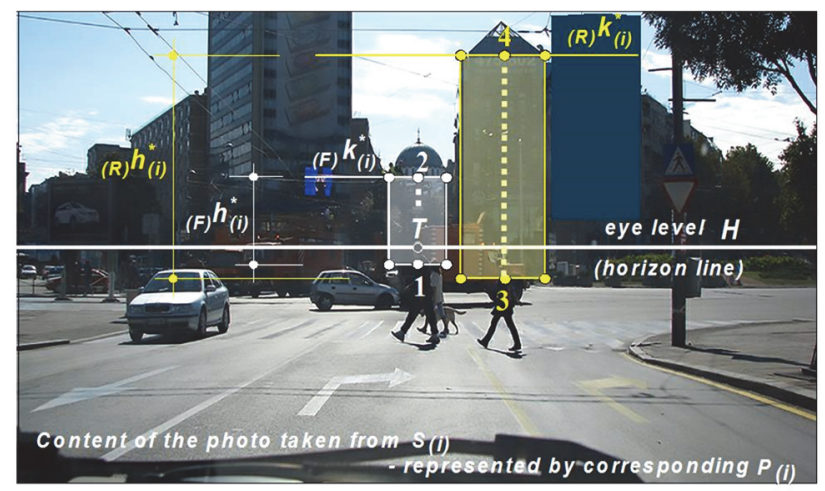

Figure 3 Enlarged arbitrarily chosen "on-path" photo that contains two sets of manually marked points $((1,2)$ and $(3,4))$ as representatives of $F$ and $R$ heights which are perceived from the corresponding $s_{(i)}$

Thus, two pairs of scene-points are modelled as illusion determinants-related: $(1,2)$ and $(3,4)$ (marked on Fig. 3). These points are arbitrarily chosen but to satisfy the following:(a) to be clearly visible/recognizable and, thus, easily selectable on the majority of photos taken (on three minimum), and (b) to be located on the corresponding masses of $F$ and $R$, bearing in mind that their connectors (dotted lines) are vertical- expressing the heights ${ }_{(F)} h_{(i)}^{*}$ and ${ }_{(R)} h_{(i)}^{*}$ of $F$ and $R$, respectively (enabling so subsequent photogrammetric restitution of the actual heights: ${ }_{F)} h^{A}$ and ${ }_{(R)} h^{A}$ ). For meanings of quoted linear dimensions see Section 3 - Fig. 1.

To solve the actual heights of $F$ and $R$ (by computing lengths of the previously defined connectors photogrammetrically), marked points are subsequently automatically referenced and photogrammetrically processed.

The chosen photogrammetric presets, including the description of the entire procedure, will not be presented here, because they do not match the scope of this research at all.

\subsubsection{Experimental Data Processing: Inputs and Outputs}

The "raw" result of performed photogrammetric processing of the $E M$ is a set of identified relative $(x, y, z)$ coordinates of both marked points and of all $S_{(i)}$ s.

To convert those relative coordinates into absolute ones, namely, to transform relative coordinate-system into the Descartes absolute coordinate-system (with $x$ - and $y$-axis positioned onto the ground/street-plane so that the direction of $x$-axis be the same as that of the street's symmetry-axis), the whole model is subsequently re- coordinated and scaled. It is done by knowing lengths of several control edges of $E F$ entities (objects) which are selected to be visible on three consecutive photos min. and to match directions of the adopted Descartes $x$ - and $y$-axis. The entire scaling procedure will neither be described further in the text, because it is also irrelevant from the subject-related point of view.

Bearing in mind the fact that camera positions were not chosen in reality to be strictly mutually equidistant, the

average distance $\bar{d}_{(S)}$ (between station-points consecutives) is adopted as invariant for clarity reason and represented as such by an arithmetic mean value of corresponding photogrammetrically solved individual $d_{\left(S_{(i)}: F\right)}$ and $d_{\left(S_{(i)}: R\right)}$ values $(i=1,10)$ (calculated using Eq. (1)).

Taking into account that the camera's movementdirection was not ideally horizontal, the average camera

height ${ }_{(S)} \bar{h}$ is also adopted as invariant and represented by an arithmetic mean value of all photogrammetrically solved individual $S_{(i)}$ heights (calculated using Eq. (2)).

So, $P R$-inputs are represented by the following parameters: (a) used control ("scaling") lengths and marked control points-pairs that correspond to those lengths (namely to their endings), and (b) two marked points-pairs signed as $(1,2)$ and $(3,4)$.

$P R$-outputs (hereinafter: $P O \mathrm{~s}$ ) are obtained values that refer to both: (a) actual heights of $F$ and $R$, including their interdistance $d_{(R: F)}$ (as final photogrammetric processing outcomes), and (b) other relevant intermediate output-data.

The list of concrete values of subject-related $P R$-outputs is shown in Tab. 1 (references to relevant calculation-wise equations are in brackets). Due to a limited number of pages, sets of photogrammetrically obtained $P R$-outputs, signed as $P O-4, \mathrm{P} O-5$ and $\mathrm{P} O-7$, are not shown in this paper.

Table 1 Concrete values of the $P R$-procedure outputs (the references to relevant calculation-wise equations are in brackets)

\begin{tabular}{|c|c|c|c|c|c|}
\hline Data-type & Id & Value & Data-type & Id & Value \\
\hline $\begin{array}{l}{ }_{(F)} h^{A} \\
/ \mathrm{m}\end{array}$ & $P O-1$ & $\begin{array}{c}70,032 \\
\left(^{*}\right)\end{array}$ & $\begin{array}{c}d_{\left(S_{(i): R)}\right.} \\
/ \mathrm{m}\end{array}$ & $P O-5$ & $(* *)$ \\
\hline $\begin{array}{l}(R) h^{A} \\
/ \mathrm{m}\end{array}$ & $P O-2$ & $\begin{array}{c}21,983 \\
\left(^{*}\right)\end{array}$ & $\begin{array}{l}\bar{d}_{(S)} \\
/ \mathrm{m}\end{array}$ & $P O-6$ & $\begin{array}{l}72,995 \\
\text { Eq. (1) }\end{array}$ \\
\hline $\begin{array}{c}d_{(R: F)} \\
/ \mathrm{m}\end{array}$ & $P O-3$ & $\begin{array}{c}450,009 \\
\left(^{*}\right)\end{array}$ & $\begin{array}{c}\left(S_{(i)}\right) h^{A} \\
/ \mathrm{m}\end{array}$ & $\mathrm{PO}-7$ & $(* *)$ \\
\hline $\begin{array}{c}d_{\left(S_{(i)}: F\right)} \\
\quad / \mathrm{m}\end{array}$ & $\mathrm{PO}-4$ & $(* *)$ & $\begin{array}{l}{ }_{(S)} \bar{h} \\
/ \mathrm{m}\end{array}$ & $P O-8$ & $\begin{array}{c}1,809 \\
\text { Eq. (2) }\end{array}$ \\
\hline${ }^{(* *)} \mathrm{Ra}$ & a pho & $\begin{array}{l}\text { nal phot } \\
\text { grammet }\end{array}$ & $\begin{array}{l}\text { rammetric } \\
\text { ally solved }\end{array}$ & $\begin{array}{l}\text { ults } \\
\text { r each }\end{array}$ & e $S_{(i)} \mathrm{s}$ \\
\hline
\end{tabular}

\subsection{AR-procedure}

\subsubsection{Fundamentals}

The $A R$-procedural stage is used to obtain intermediate and final results in the form of delta- and psi-quantifiers values - calculated for each of the $S_{(i)}$ s they correspond to. 


\subsubsection{Experimental Data Processing: Inputs and Outputs}

The $A R$-procedure inputs are adequate $P R$-procedure outputs. The $A R$-procedure outputs are calculated using $A R$-equations (3) to (7). Their concrete values are shown in Tab. 2 and Tab. 3, respectively.

Table 2 Concrete values of the AR-procedure inputs

\begin{tabular}{|c|c|c|c|c|c|}
\hline Data-type & Id & Value & Data-type & Id & Value \\
\hline $\begin{array}{l}{ }_{(F)} h^{A} \\
/ \mathrm{m}\end{array}$ & $\begin{array}{l}P O-1 \\
\rightarrow A I-1\end{array}$ & $\underset{\left({ }^{*}\right)}{70,032}$ & $\begin{array}{c}d_{\left(S_{(i)}: F\right)} \\
/ \mathrm{m}\end{array}$ & $\begin{array}{c}P O-4 \\
\rightarrow A I-4\end{array}$ & ${ }^{(* *)}$ \\
\hline $\begin{array}{l}{ }_{(R)} h^{A} \\
/ \mathrm{m} \\
\end{array}$ & $\begin{array}{c}P O-2 \\
\rightarrow A I-2\end{array}$ & $\underset{\left.{ }^{*}\right)}{21,983}$ & $\begin{array}{c}d_{\left(S_{(i): R)}\right.} \\
/ \mathrm{m}\end{array}$ & $\begin{array}{l}P O-5 \\
\rightarrow A I-5\end{array}$ & $\left({ }^{* *}\right)$ \\
\hline $\begin{array}{c}d_{(R: F)} \\
/ \mathrm{m}\end{array}$ & $\begin{array}{c}P O-3 \\
\rightarrow A I-3\end{array}$ & $\underset{\left({ }^{*}\right)}{450,009}$ & $\begin{array}{l}\bar{d}_{(S)} \\
/ \mathrm{m}\end{array}$ & $\begin{array}{c}P O-6 \\
\rightarrow A I-6\end{array}$ & 72,995 \\
\hline
\end{tabular}

Table 3 Concrete values of the AR-procedure outputs

\begin{tabular}{|c|c|c|c|c|c|}
\hline Data-type & Id & Value & Data-type & Id & Value \\
\hline $\begin{array}{c}(F) \\
/ \mathrm{m}\end{array}$ & $A O-1$ & $\begin{array}{c}68,223 \\
\text { Eq. (3) }\end{array}$ & $\begin{array}{c}\Delta_{(i)} \\
/ \circ\end{array}$ & $A O-3$ & $\begin{array}{c}\text { Eq. (5) } \\
\text { or } \\
\text { Eq. (6) }\end{array}$ \\
\hline $\begin{array}{c}{ }_{(R)} h \\
/ \mathrm{m}\end{array}$ & $A O-2$ & $\begin{array}{c}20,174 \\
\text { Eq. (4) }\end{array}$ & $\begin{array}{c}\Psi_{(i \rightarrow(i-1))} \\
/ \% / \mathrm{m}\end{array}$ & $A O-4$ & Eq. (7) \\
\hline
\end{tabular}

As both the shooting/perception direction and the ground/street-plane (common for $F$ and $R$ ) are considered nearly horizontal, only those segments of perceived linear sizes of $F$ and $R$ which are positioned above $H$-plane will be taken into account [1] (actually those expressed with the segments of the heights ${ }_{(F)} h_{(i)}^{*}$ and ${ }_{(R)} h_{(i)}^{*}$ positioned above the horizon-line of the corresponding $P_{(i)}$ and, more precisely, with their corresponding angular sizes ${ }_{\left({ }_{(F)} k\right)} \alpha_{(i)}$ and $\left.{ }_{\left.(R)^{k}\right)} \alpha_{(i)}\right)$. Accordingly, Eq. (3) and Eq. (4) are applied to calculate the first two intermediate output-data of the $A R$-procedure: $A O-1\left(_{(F)} h\right)$ and $A O-2\left(_{(R)} h\right)$.

The final $A O-3$ outputs $\left(\Delta_{(i)}\right)$ are calculated for each of the $S_{(i)}$ s by the usage of $A I-3$ to $A I-5$ as well as $A O-1$ and AO-2 (applying Eq. (5) or Eq. (6)). The final $A R$-outputs signed as $A O-4\left(\Psi_{(i \rightarrow(i-1))}\right)$ are calculated for each of the $S_{(i)}$ s by the usage of $A I-6$ and $A O-3$ (applying Eq. (7)). Their concrete values are shown in Tab. 4.

\subsubsection{Graphical Outputs Creation}

Based on the following data-sets obtained: (a) the set of ten restituted $S_{(i)}$ s positions distributed along the observer's movement-path, (b) the set of ten calculated delta-quantifiers values, and (c) the set of ten calculated psi-quantifiers values (both referred to corresponding $\left.S_{(i)} \mathrm{s}\right)$, it becomes possible to restitute the behaviour of the examined illusion - by visualizing it in forms of delta- and psi-quantifiers charts (Fig. 4).

Table 4 Concrete values $\left(\times 10^{-5}\right)$ of final $A R$-procedure outputs signed as $A O-3$ and $A O-4$ obtained in the function of camera positions $\left(S_{(i)}\right.$ S) they correspond to

\begin{tabular}{|c|c|c|c|c|c|c|c|c|c|c|c|}
\hline $\mathrm{Id} / S_{(i)}$ & $S_{(1)}$ & $S_{(2)}$ & $S_{(3)}$ & $S_{(4)}$ & $S_{(5)}$ & $S_{(6)}$ & $S_{(7)}$ & $S_{(8)}$ & $S_{(9)}$ & $S_{(10)}$ \\
\hline$A O-3$ & $-4752,500$ & $+151,527$ & $+1931,110$ & $+2683,167$ & $+3005,154$ & $+3128,390$ & $+3142,480$ & $+3106,672$ & $+3038,997$ & $+2959,697$ \\
\hline $\mathrm{Id} / S_{(i \rightarrow i-1)}$ & & $S_{(2 \rightarrow 1)}$ & $S_{(3 \rightarrow 2)}$ & $S_{(4 \rightarrow 3)}$ & $S_{(5 \rightarrow 4)}$ & $S_{(6 \rightarrow 5)}$ & $S_{(7 \rightarrow 6)}$ & $S_{(8 \rightarrow 7)}$ & $S_{(9 \rightarrow 8)}$ & $S_{(10 \rightarrow 9)}$ \\
\hline$A O-4$ & - & $+67,180$ & $+24,336$ & $+10,302$ & $+4,419$ & $+1,685$ & $+0,193$ & $-0,490$ & $-0,929$ & $-1,084$ \\
\hline
\end{tabular}

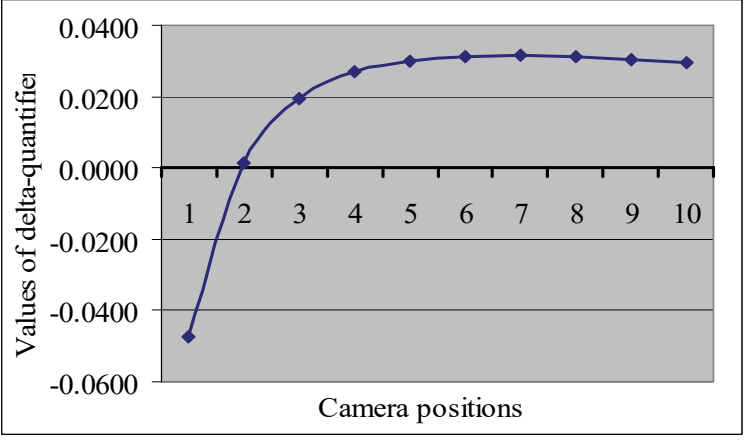

(a)

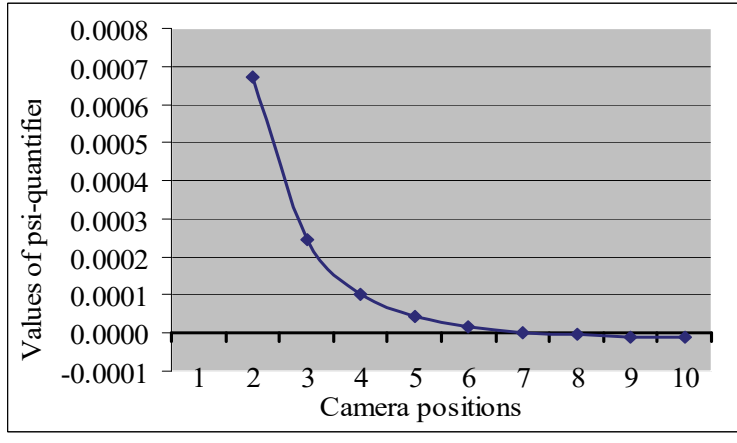

(b)

Figure 4 The behaviour-charts of the restituted illusion in the function of ten camera positions $\left(S_{(i)} s\right)$ the photos have been taken from: characteristics of delta-quantifiers distribution (a) and psi-quantifiers distribution (b)
Thus, according to global distributions of those illusion-quantifiers values, having also in mind their "plus" and "minus" sub-signs (that indicate a presence of so-called "involutory points" as important qualifiers of the entire illusion-flow), one becomes capable to read-off all of its five behavioural aspects: noticeability, appearance, duration, dynamics and overall impression [6].

By applying the subject-related technique, a global capacity of the analyzed urban-matrix's part to host the angular size-illusion is checked. Additionally, it is scientifically proven that such an ability really exists in the selected urban-matrix's part, demonstrating also the concrete behavioural characteristics of the illusion (which would be experienced as such when the observer permanently moves along Kneza Milosa Street towards St Sava Temple, if it is his major point of interest). Finally is also identified the movement-path segment (and its length) the exhibited illusion is noticeable from more significantly.

\section{ON POSSIBILITIES TO MANAGE THE RESTITUTED ILLUSION'S BEHAVIOUR}

To illustrate how to manage the restituted illusion's behaviour by changing the values of illusion-determinantsrelated data of pattern-morphogenic nature and, consequently, to control it in advance (by performing 
adequate spatial interventions that represent such changing), three experimental cases are introduced (hereinafter: ECs). They are defined below by using three fundamental/pattern-morphogenic $P R$-procedure outputdata/variables: $\quad P O-1 \quad\left({ }_{(F)} h^{A}\right), \quad P O-2 \quad\left({ }_{(R)} h^{A}\right) \quad$ and $P O-3\left(d_{(R: F)}\right)($ Tab. 5).

Table 5 Three ECs defined by using three fundamental/pattern-morphogenic PR-procedure outputs

\begin{tabular}{|c|c|c|c|}
\hline Data-type/variable & $P O-1$ & $P O-2$ & $P O-3$ \\
\hline$P O-1$ & $E C-1$ & - & - \\
\hline$P O-2$ & - & $E C-2$ & - \\
\hline$P O-3$ & - & - & $E C-3$ \\
\hline
\end{tabular}

This idea means that each of the ECs is defined so that two of the three mentioned fundamental data-types remain invariant, while the value of the third varies three times (the first value is the actual one, while the two remaining are $50 \%$ larger than that (signed with " $>$ ") and 50\% smaller (signed with " $<")$.

For example, $E C-1$ demonstrates how delta- and psi-chart change during movement along the chosen path, when the values of $P O-1$ vary (modified by the mentioned increment $(>)$ and decrement $(<)$ ), while the values of $P O-2$ and $P O-3$ remain invariant.

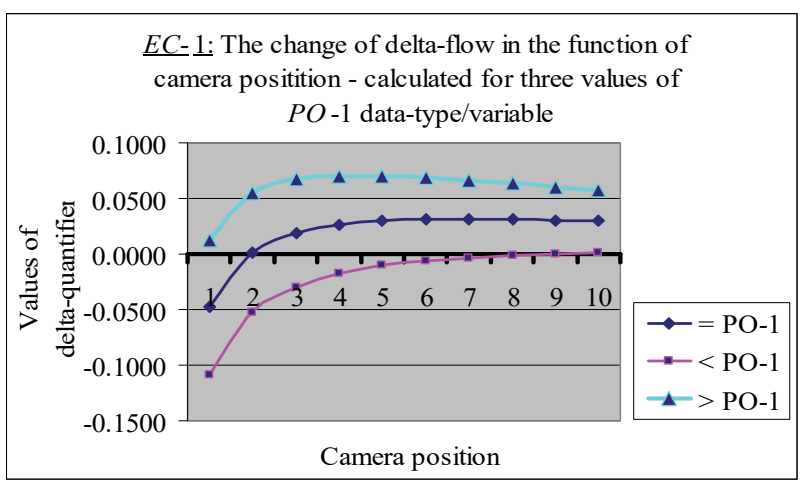

$E C-1$ : The change of psi-flow in the function of camera positition - calculated for three values of $P O-1$ data-type/variable

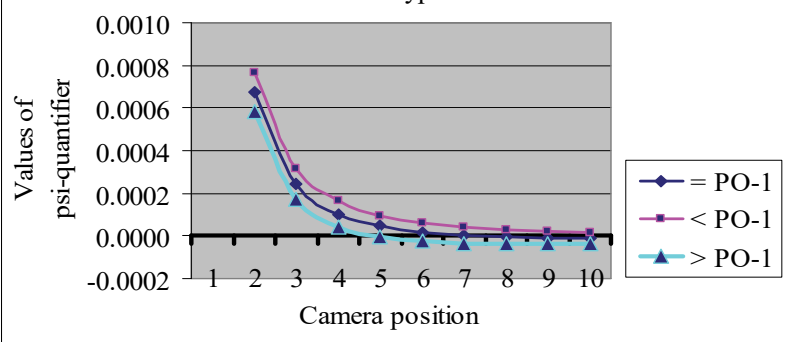

(a)

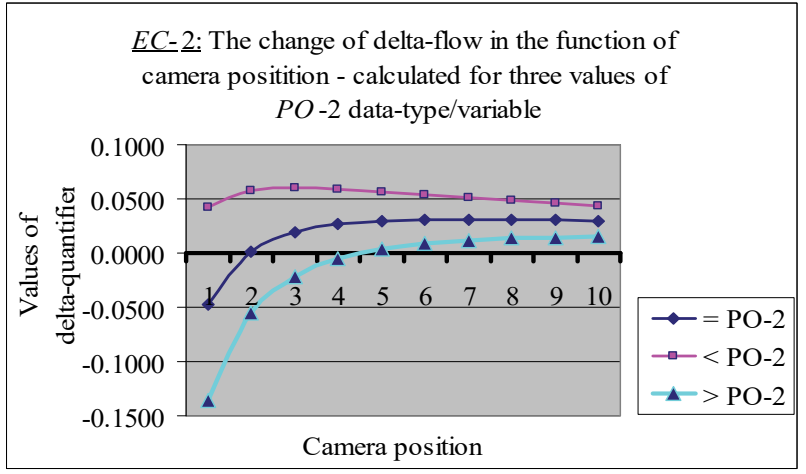

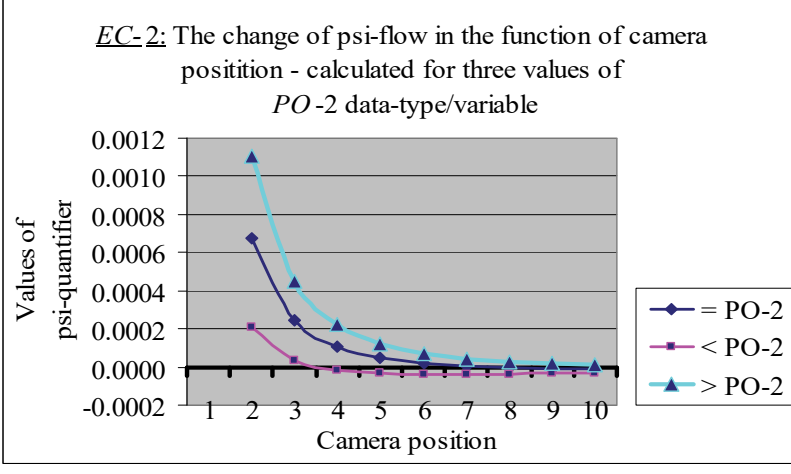

(b)

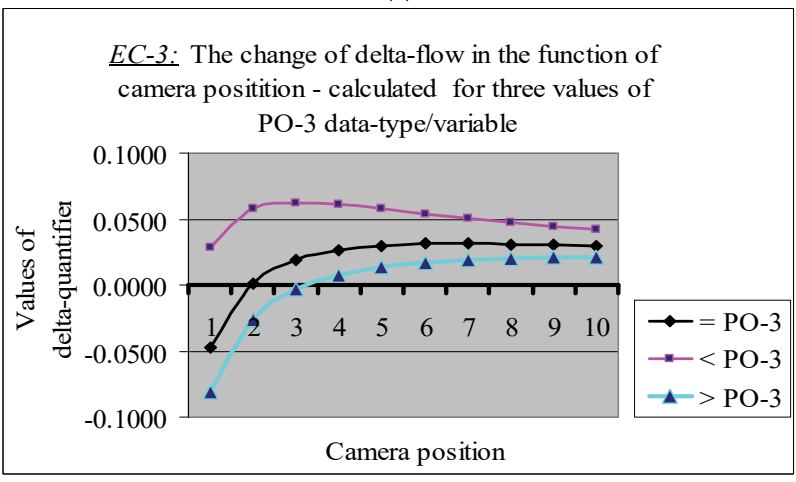

EC-3: The change of psi-flow in the function of camera positition - calculated for three values of PO-3 data-type/variable

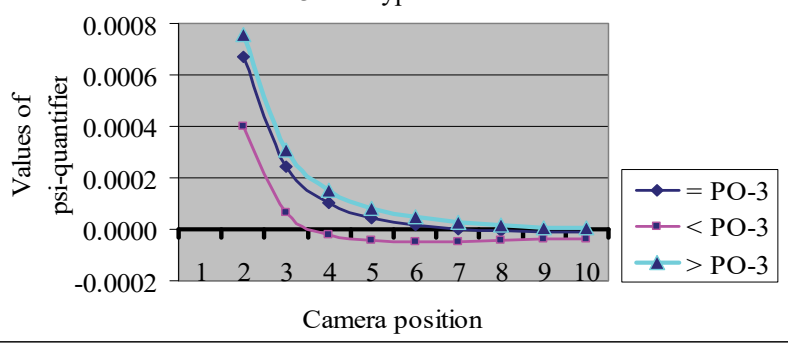

(c)

Figure $\mathbf{5}$ The variability of possible illusion-quantifiers distribution of deltaand psi-type in the function of $E C$ it refers to: (a) EC-1; (b) EC-2 and (c) EC-3

So, Fig. 5 illustrates the variability of possible illusionquantifiers distribution of delta- and psi-type in the function of $E C$ it refers to (underlying the consequences of the modified behaviour of the restituted illusion that would be noticeable during movement after applying corresponding physical interventions on $F$ and/or $R$ ).

Taking into account "behavioural variations" represented by delta- and psi-charts shown in Fig. 5 (as those expectable in reality), including the identified functional dependencies which allow to calculate (numerically model) them, by implementing this methodology it becomes possible to prevent or minimize any cause of unwanted/unpredictable visual impression's degradations of important architectural/urban structures by planning and performing adequate spatial (physical) interventions on existing/reconstructed/newly designed architectural and urban matrices.

\section{GENERAL CONCLUSION AND OUTLOOK}

The demonstrated concept of on-photo restitution of an angular size-illusion's behaviour- when manifested in existent architectural/urban spaces of simple geometry, 
allows us not only to scientifically check their capacity to host it in general, but also to modify and manage such a behaviour (as shown in Section 6).

Additionally, when the entire scene (namely all $E F$ physical entities captured by a set of taken photos) is fully photogrammetrically digitalized as a complex point-cloud (converted then into photo-realistically textured 3D-mesh, as in [27]), it is feasible to digitally simulate the illusion's behaviour in a realistic way (to estimate, programme and control it using that mesh in virtual-, especially, augmented-reality environments). With regard to that, using kinematics (broadly applicable in computer animation) might make possible to digitally simulate the observer's movement, even if she/he actually remains still at all. It could be carried out animating permanent metric transformations of relevant pattern's physical structure entities (declared focus, marker and their closer surroundings) to enable to create an expression of such an imaginary movement - emulating previously adequately set and programmed causal illusion's behaviour.

To perform these tasks practically, the major aim of the subsequent phase of the current research is to develop a general approach that will allow the following: (a) to process (from the subject-related point of view) not only architectural/urban matrices of simple geometry (as in the current/conceptual experiment), but also structures of more complex morphology (when, among others, a movement-path is not exclusively horizontal) and (b) contrary, to use the already known behaviour-charts of the "existent" illusion being exhibited in concrete architectural/urban spaces during movement, in order to restitute their pattern-morphogenic characteristics in the form of analyzed determinants-related data/variables (signed as: $P O-1, P O-2$ and $P O-3$ ).

The reverse use of the entire research results in the field of psychology will also be attainable: to simulate an illusion's appearance and its behaviour in virtual digital surroundings in order to investigate how visual perception and visual attention's mechanisms $[20,21]$ respond to a variety of positive and negative contextual signals "offered" by those surroundings when they are influenced, this time, by an exhibited angular size-illusion. Regarding the similar context, with respect to investigation [28] of how drivers react to a virtual environment when a driver simulator is used, by applying the same technology, it will also be possible to study how they would react to various behavioural aspects of the simulated illusion - exhibited while driving along the virtual paths toward the objects of interest (foci and markers), under the influence of a set of numerous illusion determinants of various kinds.

\section{Acknowledgement}

This investigation is performed at the University of Belgrade - Faculty of Architecture within the "Laboratory for Research, Valorisation, Conservation and Presentation of Cultural Heritage", founded and financed by the "Ministry of Education, Science and Technological Development" of the Republic of Serbia.

\section{REFERENCES}

[1] Djordjević, Dj. \& Vujic, G. (2010). Visual illusion of the change of the size of architectural and urban objects observed upon a change of the observer's distance: parameters that influence it phenomenological. SPATIUM International Review, 22, 38-46. https://doi.org/10.2298/SPAT1022038D

[2] Murray, S. O., Boyaci, H., \& Kersten, D. (2006). The representation of perceived angular size in human primary visual cortex. Nature Neuroscience, 9, 429-434. https://doi.org/10.1038/nn1641

[3] McCready, D. (1965). Size-distance perception and accommodation - convergence micropsia: A critique. Vision Research, 5(4-5), 189-206. https://doi.org/10.1016/0042-6989(65)90065-9

[4] Komoda, M. K. \& Ono, H. (1974). Oculomotor adjustments and size-distance perception. Perception \& Psychophysics, 15, 353-360. https://doi.org/10.3758/BF03213958

[5] Ono, H., Muter, P., \& Mitson, L. (1974). Size-distance paradox with accommodative micropsia. Perception \& Psychophysics, 15, 301-307. https://doi.org/10.3758/BF03213948

[6] Djordjevic, Dj. \& Djukanovic, G. (2017). The impact of various architectural/urban patterns on the behaviour of an angular size-illusion - noticeable during the observer's movement. METU Journal of the Faculty of Architecture, 34(1), 21-41. https://doi.org/10.4305/METU.JFA.2017.1.10

[7] McCready, D. (1986). Moon illusions redescribed. Perception \& Psychophysics, 39, 64-72. https://doi.org/10.3758/BF03207585

[8] Restle, F. (1970). Moon illusion explained on the basis of relative size. Science, 167(3921), 1092-1096. https://www.jstor.org/stable/1728666

[9] Kilpatrick, F. P. \& Ittelson, W. H. (1953). The size-distance invariance hypothesis. Psychological Review, 60(4), 223231. https://doi.org/10.1037/h0060882

[10] Hastorf, A. H. (1950). The influence of suggestion on the relationship between stimulus size and perceived distance. Journal of Psychology, 29(1), 195-217. https://doi.org/10.1080/00223980.1950.9712784

[11] Bolles, R. C. \& Bailey, D. E. (1956). Importance of object recognition in size constancy. Journal of Experimental Psychology, 51(3), 222-225. https://doi.org/10.1037/h0048080

[12] Rock, I. \& McDermott, W. (1964). The perception of visual angle. Acta Psychologica, 22(2), 119-134. https://doi.org/10.1016/0001-6918(64)90011-3

[13] McCready, D. (1985). On size, distance and visual angle perception.Perception \& Psychophysics, 37, 323-334. https://doi.org/10.3758/BF03211355

[14] Colheart, M. (1970). The effect of verbal size information upon visual judgments of absolute distance. Perception \& Psychophysics, 9, 222-223. https://doi.org/10.3758/BF03212634

[15] Foley, J. M. (1980). Binocular distance perception.Psychological Review, 87(5), 411-434. https://doi.org/10.1037/0033-295X.87.5.411

[16] Roscoe, S. N. (1984). Judgments of size and distance with imaging displays. Human Factors, 26(6), 617-629. https://doi.org/10.1177/001872088402600601

[17] Higashiyama, A. (1992). Anisotropic perception of visual angle: Implications for the horizontal-vertical illusion, over constancy of size, and the moon illusion. Perception \& Psychophysics, 51, 218-230. https://doi.org/10.3758/BF03212248

[18] Higashiyama, A. \& Shimono, K. (1994).How accurate is size and distance perception for very far terrestrial objects? Perception \& Psychophysics, 55, 429-442. https://doi.org/10.3758/BF03205300

[19] Gogel, W. C. \& Eby, D. W. (1997). Measures of perceived linear size, sagittal movement, and visual angle from optical 
expansions and contractions. Perception \& Psychophysics, 59, 783-806. https://doi.org/10.3758/BF03206024

[20] Milosevic, S. (2002). Percepcija, paznja i motorna aktivnost. Zavod za udzbenike i nastavna sredstva.

[21] Lee, S., Cinn, E., Jin, Y., \& Jung, J. (2015). Using an eye tracker to study three-dimensional environmental aesthetics: the impact of architectural elements and educational training on viewers' visual attention. Journal of Architectural and Planning Research, 32(2), 145-167. https://www.jstor.org/stable/44113105

[22] Abu-Obeid, N. \& Abu-Safieh, S. (2010). The effect of the angle between navigation paths and transitional spaces on spatial knowledge. Journal of Architectural and Planning Research, 27(1), 57-68. https://www.jstor.org/stable/43030892

[23] Nikolić, V., Radović, Lj., Nikolić, O. Marković, B., Mitković, P., Mitković, M., \& Đurić, J. (2016). Proportional ratios and geometrical setups for achieving the hovering effect of architectonic structures. Technical Gazette Tehnički vjesnik, 23(2), 579-588. https://doi.org/10.17559/TV-20140628181558

[24] Eos Systems Inc., PhotoModeler Premium User's Manual. Retrieved from https://www.photomodeler.com/downloads/ OnlineHelp/index.html

[25] Lopez, J. T. C. (2011). Fotogrametria practica. Tantin.

[26] Deng, G. \& Falg, W. (2001). An evaluation of an off-theshelf digital close-range photogrammetric software package. Photogrammetric Engineering and Remote Sensing, 67(2), 227-233.

[27] Pejić, P., Krasić, S., \& Dragović, M. (2017). 3D virtual modelling of existing objects by terrestrial photogrammetric methods - case study of barutana. Technical Gazette Tehnički vjesnik, 24(1), 233-239. https://doi.org/10.17559/TV-20141018155354

[28] Maghelal, P., Natesan, P., Naderi R. J., \& Kweon, B. S. (2011). Investigating the use of virtual reality for pedestrian environments. Journal of Architectural and Planning Research, 28(2), 104-117.

https://www.jstor.org/stable/43030932

\section{Contact information:}

Djordje DJORDJEVIC, PhD, Assistant Professor

(Corresponding author)

University of Belgrade, Faculty of Architecture, Department of Architecture,

73/2 Kralja Aleksandra Blvd., Belgrade 11000, Serbia

E-mail: djordje@arh.bg.ac.rs

Mirjana DEVETAKOVIC RADOJEVIC, PhD, Assistant Professor

University of Belgrade, Faculty of Architecture, Department of Architecture,

73/2 Kralja Aleksandra Blvd., Belgrade 11000, Serbia

E-mail: mirjana.devetakovic@arh.bg.ac.rs

Nikola POPOVIC, Assistant

University of Belgrade, Faculty of Architecture, Department of Architecture,

73/2 Kralja Aleksandra Blvd., Belgrade 11000, Serbia

E-mail: nikola.popovic.bk@arh.bg.ac.rs 